\title{
The effects of aging and maternal protein restriction during lactation on thymic involution and peripheral immunosenescence in adult mice
}

\author{
Chantal A. A. Heppolette ${ }^{1}$, Jian-Hua Chen ${ }^{1}$, Sarah K. Carr ${ }^{1}$, Donald B. Palmer ${ }^{2}$ and \\ Susan E. Ozanne ${ }^{1}$ \\ ${ }^{1}$ University of Cambridge Metabolic Research Laboratories and MRC Metabolic Diseases Unit, Wellcome Trust-MRC Institute \\ of Metabolic Science, Addenbrooke's Hospital, Cambridge, UK \\ ${ }^{2}$ Department of Comparative Biomedical Sciences, Royal Veterinary College, University of London, London, UK \\ Correspondence to: Chantal A. A. Heppolette, email: ch1107@ic.ac.uk \\ Keywords: developmental programming, immunosenescence, lifespan, maternal diet, thymic involution, Gerotarget \\ Received: September 21, 2015 Accepted: January 25, 2016 \\ Published: February 03, 2016
}

\section{ABSTRACT}

Environmental factors such as nutrition during early life can influence long-term health, a concept termed developmental programming. Initial research was focused towards the effects on metabolic health but more recent studies have demonstrated effects on parameters such as lifespan and immunity. In this study we report that maternal protein restriction during lactation in mice, that is known to prolong lifespan, slows aging of the central and peripheral immune systems. Offspring of dams fed a postnatal low-protein (PLP) diet during lactation had a significant increase in thymic cellularity and $T$ cell numbers across their lifespan compared to controls, and a less marked age-associated decrease in thymocyte cluster of differentiation (CD) 3 expression. PLP animals also demonstrated increased relative splenic cellularity, increased naïve: memory $\mathrm{CD4}^{+}$and $\mathrm{CD8}^{+} \mathrm{T}$ cell ratios, increased staining and density of germinal centres, and decreased gene expression of p16 in the spleen, a robust biomarker of aging. A slower rate of splenic aging in PLP animals would be expected to result in decreased susceptibility to infection and neoplasia. In conclusion nutritionally-induced slow postnatal growth leads to delayed aging of the adaptive immune system, which may contribute towards the extended lifespan observed in these animals.

\section{INTRODUCTION}

The developmental origins of health and disease hypothesis describes the phenomenon whereby exposures in utero and early postnatal life modulate long-term health [1]. Original studies focused on how early life events increase the susceptibility to metabolic diseases such as type 2 diabetes and cardiovascular disease [2-4]. However accumulating evidence suggests a role for maternal exposures on non-metabolic disease parameters such as psychiatric disorders, osteoporosis and immune health [5-7]. Interventions can also be beneficial, evidenced by numerous studies showing that the intake of breast milk significantly reduces the risk of developing metabolic syndrome in later life [8].

The development of the adaptive immune system in mammals occurs during fetal and early postnatal growth periods, during which they are vulnerable to environmental insults [9]. Epidemiological data from rural Gambia have shown that infants born during the hungry season where food supplies are limited, have a smaller thymic volume and are more prone to infection compared to those born in the harvest season $[6,10]$. Another study in humans observed that a longer period of breastfeeding increased thymic volume in infants, which is indicative of increased immune capacity [11]. Studies using animal models also support the concept that early nutrition has an effect on the immune system. For example, Osgerby and co-workers observed reduced thymic mass in fetal sheep at various stages of gestation in underfed mothers [12]. Previous work in our laboratory revealed that offspring born to normally fed dams and suckled by protein restricted dams (postnatal low protein: PLP offspring), demonstrated slow growth during lactation, extended longevity and continued 
Table 1: Effect of maternal diet on body weight during lifespan

\begin{tabular}{|c|c|c|}
\hline Age & Diet & Mean body weight / g \\
\hline \multirow{2}{*}{$21 \mathrm{~d}$} & Control & $7.96 \pm 0.23$ \\
\cline { 2 - 3 } & PLP & $6.43 \pm 0.27 * * *$ \\
\hline \multirow{2}{*}{$3 \mathrm{~m}$} & Control & $28.14 \pm 0.50$ \\
\cline { 2 - 3 } & PLP & $26.52 \pm 0.40 * *$ \\
\hline \multirow{2}{*}{$18 \mathrm{~m}$} & Control & $44.90 \pm 1.30$ \\
\cline { 2 - 3 } & PLP & $41.66 \pm 2.30 *$ \\
\hline \multirow{2}{*}{$23 \mathrm{~m}$} & Control & $41.11 \pm 1.90$ \\
\cline { 2 - 3 } & PLP & $33.70 \pm 5.70 * *$ \\
\hline
\end{tabular}

Body weights of offspring were measured weekly during their lifespan and were consistently lower in the PLP group compared to control animals at $21 \mathrm{~d}(* * * p<0.001), 3 \mathrm{~m}(* * p<0.01), 18 \mathrm{~m}(* p<0.05)$ and $23 \mathrm{~m}$ $(* * p<0.01) . \mathrm{n}=16-30$ per group. Data are represented as mean $+/-$ SEM.

growth of the thymus between 21 days and 3 months (which was not apparent in control animals) [13, 14].

An efficient and functional adaptive immune system is considered vital for protection against infection, autoimmune disease and tumour growth, and comprises of $\mathrm{T}$ and $\mathrm{B}$ lymphocytes, which originate in the thymus and bone marrow respectively. In thymocyte development, stem cells derived from bone marrow enter the thymus via the cortico-medullary junction (CMJ) where they commit to the $\mathrm{T}$ cell fate. These thymocytes which are double negative (DN) for both co-receptors CD4 and CD8, migrate to the cortex where they progress through four developmental stages characterised by the expression of CD25 and CD44: DN1 (CD44+ CD25-), DN2 (CD44 ${ }^{+}$ CD25 ${ }^{+}$, DN3 (CD44- ${ }^{-}$25 $\left.5^{+}\right)$and DN4 (CD44- ${ }^{-}$25 $\left.5^{-}\right)$ [15]. The magnitude of $\mathrm{T}$ cell receptor (TCR identified by CD3 marker) expression increases on cells throughout thymocyte development [16]. Co-receptor expression is then upregulated forming double positive (DP) $\mathrm{CD}^{+}$ $\mathrm{CD}^{+}$thymocytes [17] and subsequently these cells differentiate into either single positive (SP) $\mathrm{CD}^{+}$or $\mathrm{SP}$ $\mathrm{CD}^{+}$thymocytes, which migrate to the medulla and are released into the periphery [18]. Newly developed T and B lymphocytes migrate to secondary lymph nodes such as the spleen, whereby following activation, they initiate an effector immune response.

The immune system becomes impaired with increasing age, which is termed immunosenescence, and accounts for the increase in immune related diseases observed in the elderly [19]. One of the most notable changes is the involution of the thymus which occurs with increasing age, and is characterized by a reduction in size, epithelial space and cellularity. Together with a disorganized microenvironment, this results in a reduction in T cell output [20]. Such alterations in the thymus lead to a reduction in number and diversity of naïve $\mathrm{T}$ cells in the periphery, leading to an age-associated increase in memory $\mathrm{T}$ cells [21]. These characteristics directly contribute towards the increased susceptibility to infection and neoplasia in the elderly population. Therefore environmental exposures during early life that can delay the development of immunosenescence are likely to have large implications for the healthspan as well as the lifespan of an individual.

In the current study we tested the hypothesis that immunosenescence can be influenced by early life events. We proposed that animals exposed to a maternal low protein diet during lactation, which are known to have an increased lifespan, would demonstrate delayed aging of the immune system compared to control offspring. To address this hypothesis we studied several immune parameters across the lifespan in PLP and control mice. The effect of a PLP diet was analyzed in both the thymus and spleen, two key immune tissues.

\section{RESULTS}

\section{Body weight}

The body weight of pups was recorded from birth and throughout their lifespan. Pups suckled by dams fed a PLP diet grew slowly during the lactation period and were significantly smaller compared to control offspring from day 7 onwards $(p<0.001)$ (Figure 1) as described previously [22]. This difference in body weight was maintained throughout their lifespan (Table 1). 
Figure 1: Effect of maternal diet on body weight during lactation. Body weights of offspring were measured at day 3, 7, 14 and 21 and were consistently lower from day 7 onwards in the PLP group $(* * * p<0.001)$ compared to control animals. $n$ $=16-30$ per group. Data are represented as mean $+/-$ SEM.

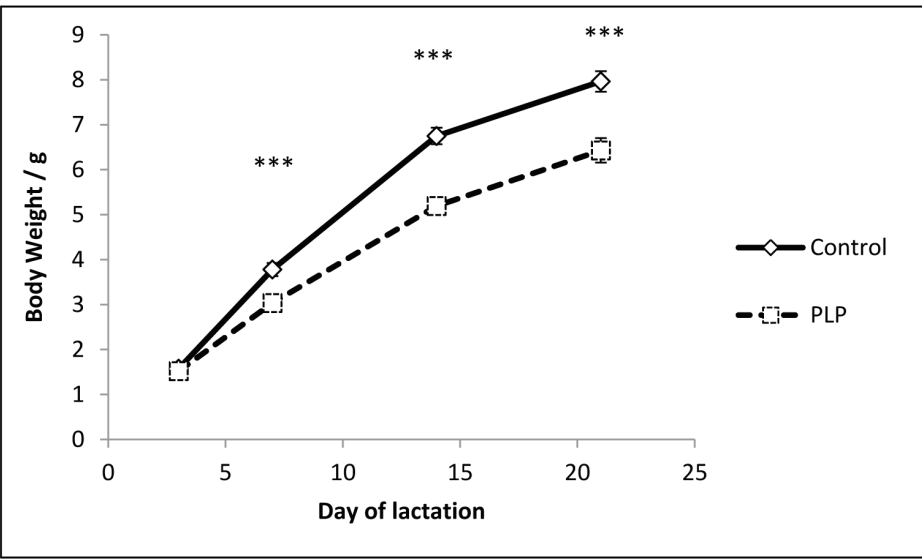

B

A

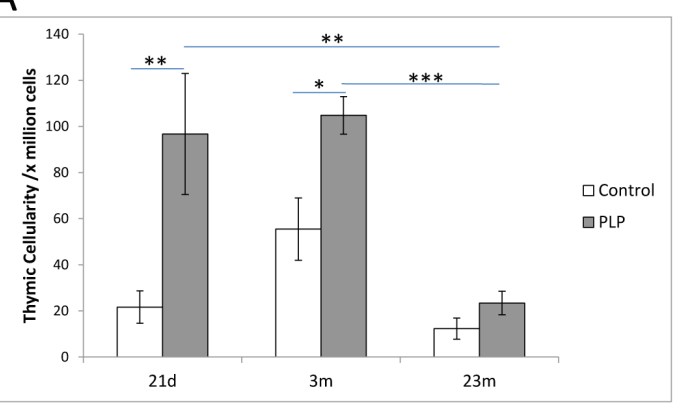

C

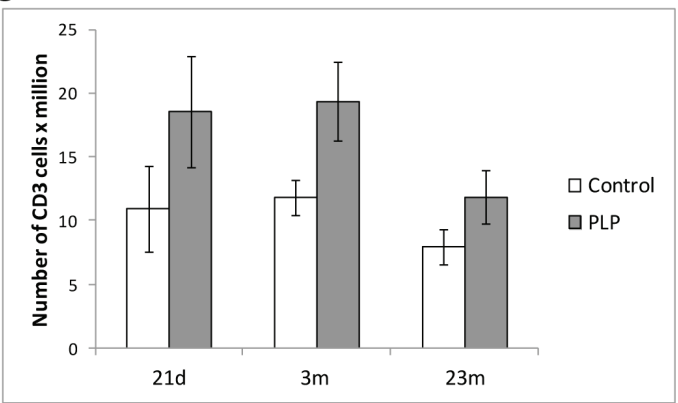

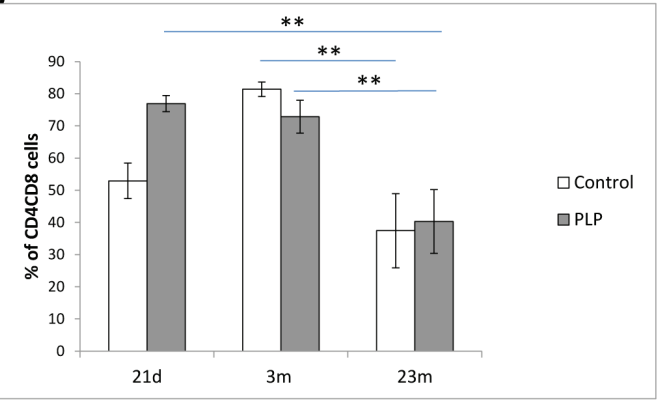

D

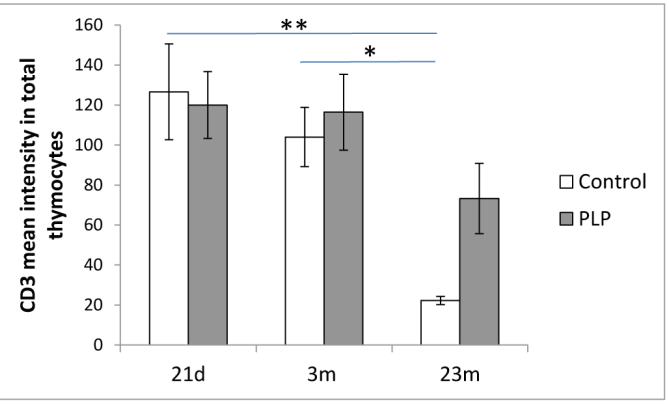

E

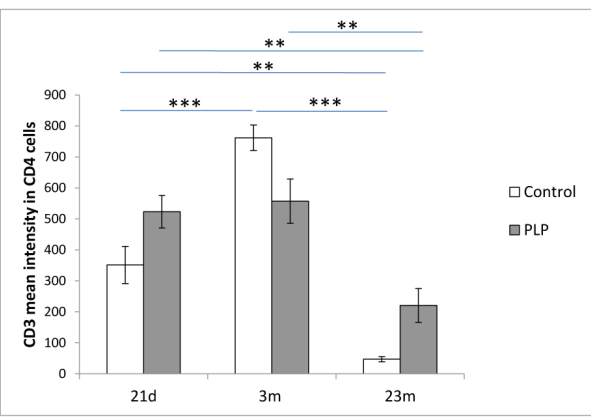

Figure 2: Effect of age and maternal diet on thymic cellularity and development. A. Thymic cellularity was significantly affected by age $(p<0.001)$. PLP offspring had significantly higher thymic cellularity compared to control animals $(p<0.001)$ especially at $21 \mathrm{~d}$ and $3 \mathrm{~m}$. B. The proportion of DP $\mathrm{CD} 4^{+} \mathrm{CD} 8^{+}$cells as measured by flow cytometry, significantly decreased with age $(p<0.001)$. However there was no effect of maternal diet. C. The number of $\mathrm{CD}^{+}$thymocytes were significantly higher in PLP offspring compared to control animals $(p<0.05)$. However there was no significant effect of age on these cells. D. The density of CD3 expression on total thymocytes decreased significantly with age $(p<0.01)$. E. The level of CD3 expression in CD4 $4^{+}$cells peaked at $3 \mathrm{~m}$, and decreased by $23 \mathrm{~m}$ of age $(p<0.001)$. However this change with age was less substantial in PLP animals $(p<0.05) .{ }^{*} p<0.05,{ }^{* *} p<0.01,{ }^{* * *} p<0.001 . n=$ 5-9 per group. Data are represented as mean +/- SEM. 


\section{Thymic cellularity}

Concurrent to previous findings, thymic cellularity was significantly affected by age $(p<0.001)$ (Figure 2A) [20]. Given that our previous study demonstrated an effect of maternal diet on thymic weight, we further investigated the effect of maternal diet on thymic cellularity [13]. Here we observed that thymic cellularity was significantly higher in PLP animals compared to the control group (effect of maternal diet $p<0.001$ ) especially at $21 \mathrm{~d}$ and $3 \mathrm{~m}$ of age (Figure $2 \mathrm{~A}$ ).

\section{Thymocyte subsets}

Given the changes observed in thymic cellularity, the $T$ cell population was analysed in more detail. Using CD4 and CD8, to identify the major subsets, we found that there was no significant effect of maternal diet on the proportion of $\mathrm{DN}, \mathrm{DP}$, and $\mathrm{SP} \mathrm{CD} 4^{+}$and $\mathrm{SP} \mathrm{CD} 8^{+}$ thymocytes (data not shown). However the proportion of DP $\mathrm{CD} 4^{+} \mathrm{CD} 8^{+}$thymocytes significantly decreased with age $(p<0.001)$ (Figure 2B). Next we further analysed the $\mathrm{DN}$ subset populations and found that although they were unaffected by maternal diet, the proportion of $\mathrm{DN}$ subset cells were significantly affected by age (Table 2$)$. The proportion of DN1 cells was greatest at $23 \mathrm{~m}(p<0.001)$ whereas the proportion of DN2 cells decreased with age $(p<0.001)$. In contrast the proportion of DN3 cells was unaffected by age, but the proportion of DN4 cells peaked at $3 \mathrm{~m}$ of age $(p<0.05)$.

\section{CD3 expression on developing thymocytes}

CD3 expression on thymocytes gradually increases as they progress through this developmental pathway, giving reason to measure the mean intensity of CD3 expression on several cellular populations [16]. We observed that the number of $\mathrm{CD}^{+}$thymocytes was significantly higher $(p<0.05)$ in the PLP group compared to the control group (Figure 2C). The level of CD3 expression on thymocytes decreased between $3 \mathrm{~m}$ and $23 \mathrm{~m}$ of age in both groups $(p<0.01)$ (Figure 2D). However, the rate of decline was markedly different; with CD3 expression levels decreasing by 5-7 fold in control mice, whereas in PLP this was barely 2-fold; thereby highlighting the impact of maternal diet on CD3 expression (Figure 2D). This age-associated decrease in CD3 expression was observed in all major thymocyte subsets (data not shown). However in $\mathrm{CD}^{+}$thymocytes the age associated decrease in the expression of the CD3 marker $(p<0.001)$ was significantly less substantial in the PLP group $(p<0.05)$ compared to the control group, again suggesting a protection from age-associated changes (Figure 2E).

\section{Splenic cellularity}

Given our observation of the effect of age and maternal diet on the thymus, an investigation into the effect on a secondary lymphoid organ was undertaken. Relative splenic cellularity peaked at $3 \mathrm{~m}$ of age and decreased again by $23 \mathrm{~m}$ of age which is concurrent with previous findings [23]. It was significantly higher in the PLP group (effect of maternal diet $p<0.05$ ) compared to the control group especially at $3 \mathrm{~m}$ of age (Figure 3 ). Consistent with previous observations in rodents, splenic weight increased gradually with age but there was no significant effect of maternal diet on this parameter (data not shown) [24]. The total splenocyte $\mathrm{T}$ and B cell populations were quantified but were not significantly affected by maternal diet (data not shown).

\section{Splenic naive and memory $\mathrm{CD4}^{+}$and $\mathrm{CD8}^{+} \mathrm{T}$ cells}

The observation that PLP animals showed increased relative splenic cellularity compared to control animals may indicate that their spleens demonstrate delayed aging. The splenocyte population was therefore examined on a closer level. Given that the density of splenocytes was very low at $21 \mathrm{~d}$, further analysis was only possible at the later time points. The effect of age and maternal diet on naive and memory $\mathrm{T}$ cell populations was quantified. The percentage of naive $\mathrm{CD}^{+}(p<0.01)$ and $\mathrm{CD} 8^{+}(p<$ $0.05) \mathrm{T}$ cells was significantly higher in the PLP group compared to the control group especially at $3 \mathrm{~m}$ of age (Figure 4A and 4B). As expected the percentage of both naive $\mathrm{CD} 4^{+}$and $\mathrm{CD} 8^{+}$cells decreased significantly with age $(p<0.001)$. The proportion of memory $\mathrm{CD} 4^{+} \mathrm{T}$ cells increased significantly with age $(p<0.001)$ although this population was unaffected by maternal diet (Figure 4C). The proportion of memory $\mathrm{CD} 8^{+} \mathrm{T}$ cells was unaffected by both maternal diet and age (Figure 4D). Overall, these observations meant that the naïve:memory ratio of both $\mathrm{CD}^{+}$and $\mathrm{CD}^{+}$splenic $\mathrm{T}$ cell were significantly higher in the PLP group $(p<0.05)$ compared to the control group especially at $3 \mathrm{~m}$ of age (Figure $4 \mathrm{E}$ and $4 \mathrm{~F}$ ). As predicted the ratio of naive:memory $\mathrm{CD} 4^{+}$and $\mathrm{CD} 8^{+}$splenic $\mathrm{T}$ cells significantly decreased with age $(p<0.001$ and $p<0.01$ respectively). Altogether these results suggest that PLP animals maintain a greater naive $T$ cell population in young and old age compared to control animals.

\section{Splenic germinal centre (GC) immunohistochemistry}

In order to determine the effect of age and maternal diet on B cell activity, staining of GCs was performed (Figure 5A). PLP animals had a significantly $(p<0.05)$ higher relative number of GCs per section of spleen compared to control animals especially at $3 \mathrm{~m}$ of age 
Table 2: Effect of age and maternal diet on the proportion of DN subset thymocytes

\begin{tabular}{|l|l|l|l|l|l|}
\hline \multirow{2}{*}{ Age } & Diet & $\%$ DN1 cells & $\%$ DN2 cells & $\%$ DN3 cells & $\%$ DN4 cells \\
\hline \multirow{3}{*}{$21 \mathrm{~d}$} & Control & $36 \pm 2 \mathrm{~A}$ & $40 \pm 3 \mathrm{E}$ & $11 \pm 2$ & $12 \pm 3 \mathrm{~J}$ \\
\cline { 2 - 7 } & PLP & $22 \pm 3 \mathrm{C}$ & $39 \pm 5 \mathrm{G}$ & $12 \pm 2$ & $26 \pm 7$ \\
\hline \multirow{3}{*}{$3 \mathrm{~m}$} & Control & $24 \pm 2 \mathrm{~B}$ & $18 \pm 1 \mathrm{~F}$ & $16 \pm 3$ & $42 \pm 5$ \\
\cline { 2 - 7 } & PLP & $26 \pm 5 \mathrm{D}$ & $24 \pm 2 \mathrm{H}$ & $16 \pm 3$ & $34 \pm 5$ \\
\hline \multirow{2}{*}{$23 \mathrm{~m}$} & Control & $57 \pm 5$ & $6 \pm 2$ & $11 \pm 3$ & $27 \pm 0$ \\
\cline { 2 - 7 } & PLP & $54 \pm 8$ & $9 \pm 3$ & $12 \pm 3$ & $25 \pm 5$ \\
\hline
\end{tabular}

Thymocytes were stained with appropriate antibodies and were gated for CD4-CD8- Subsequently the proportion of DN1 (CD44+ CD25-), DN2 (CD44+ CD25+), DN3 (CD44- CD25 $)$ and DN4 (CD44CD25-) cells were analysed on the BD FACSCalibur flow cytometer. The proportion of DN1 $(p<0.001)$, DN2 $(p<0.001)$, and DN4 $(p<0.05)$ thymocytes varied with age but were not affected by maternal diet. Following a Duncan's post-hoc analysis the proportion of DN1 cells in control animals was higher at $23 \mathrm{~m}$ compared to $21 \mathrm{~d}(\mathrm{~A}$ where $\mathrm{p}<0.05)$ and $3 \mathrm{~m}$ (B where $p<0.001)$. The proportion of DN1 cells in PLP animals was also higher at $23 \mathrm{~m}$ compared to $21 \mathrm{~d}(\mathrm{C}$ where $p<0.001)$ and $3 \mathrm{~m}$ (D where $p<0.01)$. However the proportion of DN2 cells decreased with age $(p<0.001)$. In control animals the proportion of DN2 cells was lower at $3 \mathrm{~m}$ compared to $21 \mathrm{~d}$ (E where $p<0.001$ ) and was lower at $23 \mathrm{~m}$ compared to $3 \mathrm{~m}$ ( $\mathrm{F}$ where $\mathrm{p}<0.05)$. The proportion of DN2 cells in PLP animals similarly was lower at $3 \mathrm{~m}$ compared to $21 \mathrm{~d}(\mathrm{G}$ where $\mathrm{p}<0.05)$ and was lower at $23 \mathrm{~m}$ compared to $3 \mathrm{~m}$ (H where $p<0.01)$. The proportion of DN3 cells was unaffected by age, and the proportion of DN4 thymocytes peaked at $3 \mathrm{~m}(p<0.05)$. In control animals the proportion of DN4 cells was higher at $3 \mathrm{~m}$ compared to $21 \mathrm{~d}(\mathrm{~J}$ where $p<0.01)$. $\mathrm{n}=5-9$ per group. Data are represented as mean +/- SEM.

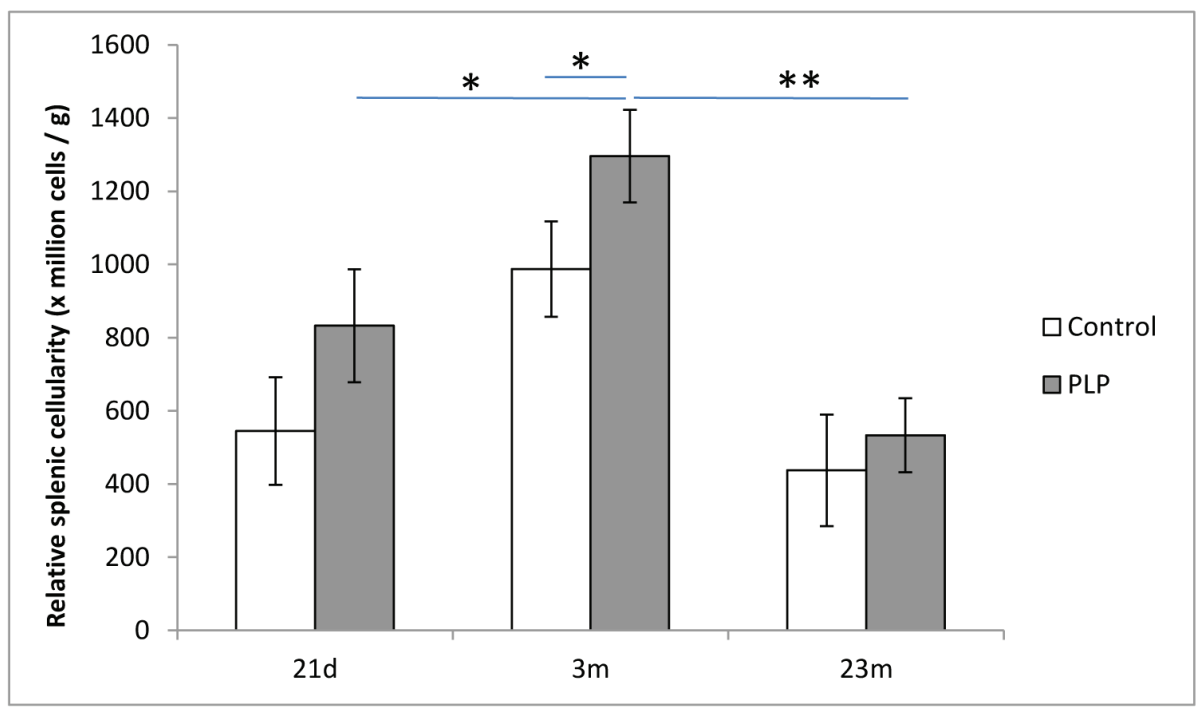

Figure 3: Relative splenic cellularity in aging PLP and control mice. In order to detect the effect of age and maternal diet on splenic involution, splenic cellularity was quantified using a Countess Automated Cell counter (Invitrogen, Paisley, UK) and represented in relation to splenic weight. Relative splenic cellularity decreased significantly in old age $(p<0.01)$ and was significantly higher in PLP offspring compared to control animals $(p<0.05) .{ }^{*} p<0.05, * * p<0.01 . n=5-9$ per group. Data are represented as mean $+/$ - SEM. 
(Figure 5B). The fluorescence intensity of GCs was also significantly $(p<0.01)$ higher in the PLP group compared to the control group especially at $18 \mathrm{~m}$ of age (Figure $5 \mathrm{C}$ ). There was however no statistically significant effect of age on these parameters. Taken together these results suggest that PLP animals have greater GC activity compared to controls.

\section{Splenic gene expression of p16}

Following our histological findings, splenic aging was investigated on a closer level. RNA levels of p16, a robust biomarker of aging, were quantified by RT-PCR.
Gene expression of p16 was significantly affected by both age $(p<0.001)$ and maternal diet $(p<0.001)$. As expected RNA levels of p16 increased with age but were also found to be significantly lower in PLP animals compared to control animals (Figure 6). In addition there was a less marked increased with age in these PLP animals compared to controls $(p<0.05)$.

\section{DISCUSSION}

The thymus is the primary site for development of immunocompetent $\mathrm{T}$ cells. In contrast the spleen is a secondary lymph organ that houses both $\mathrm{T}$ and $\mathrm{B}$
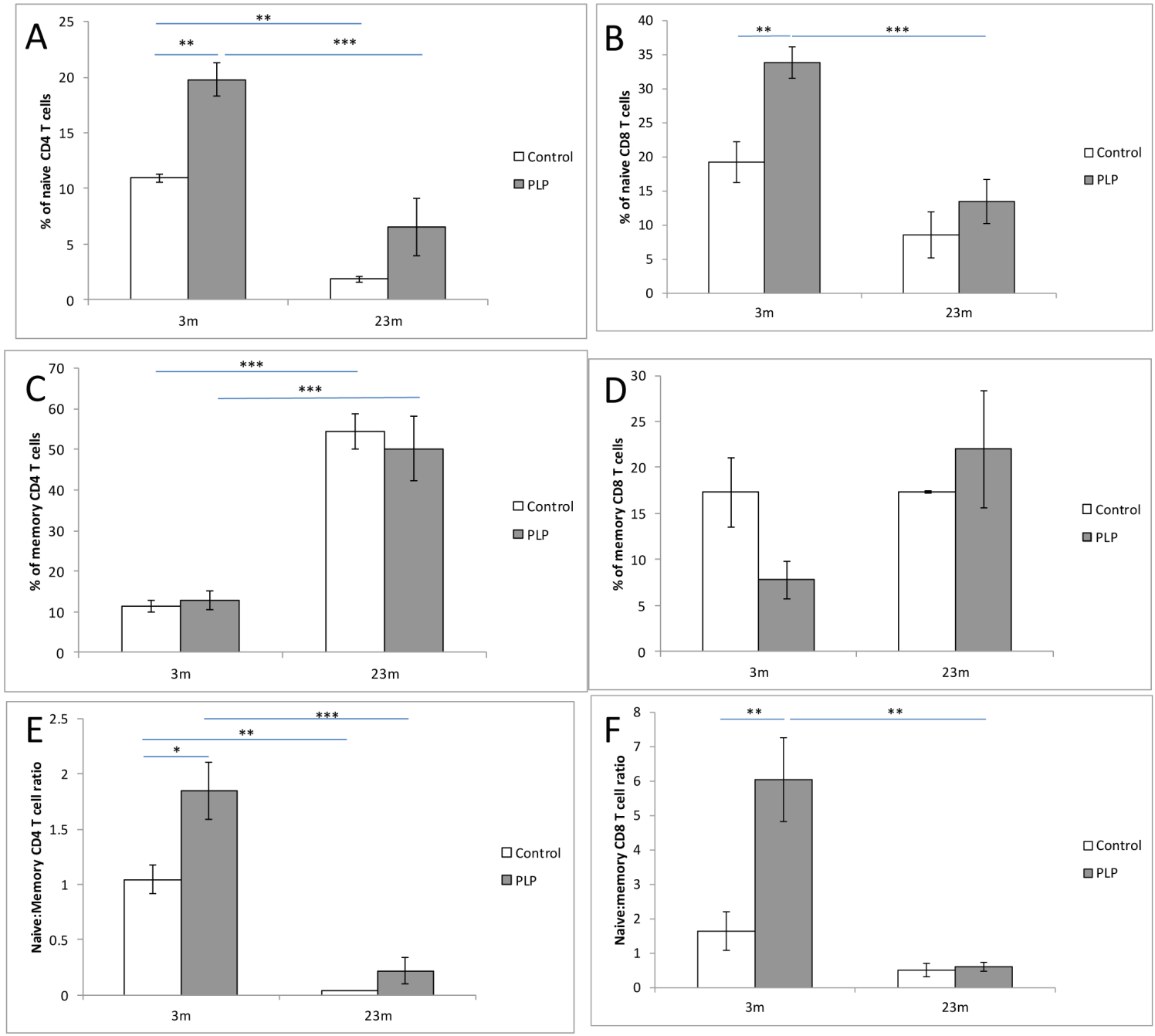

Figure 4: The impact of age and maternal diet on splenic T cells. Splenocytes were stained with appropriate antibodies. Cells were gated for either $\mathrm{CD}^{+}$or $\mathrm{CD}^{+}$and subsequently the proportion of naive cells $\left(\mathrm{CD} 62 \mathrm{~L}^{+} \mathrm{CD}^{-} 4^{-}\right)$and memory cells $\left(\mathrm{CD} 62 \mathrm{~L}^{-} \mathrm{CD} 44^{+}\right)$ cells were analysed on the BD FACSCalibur flow cytometer. The proportion of naive CD4 ${ }^{+}$cells $\mathbf{A}$. and CD $8^{+}$cells $\mathbf{B}$. was significantly affected by maternal diet ( $p<0.01$ and $p<0.05$ respectively), and was significantly higher in PLP animals compared to controls, especially at $3 \mathrm{~m}$ of age $(* * p<0.01)$. The proportion of naive $\mathrm{CD} 4^{+}$cells and $\mathrm{CD} 8^{+}$cells significantly decreased with age $(p<0.001)$. The percentage of memory $\mathrm{CD}^{+}$cells $\mathbf{C}$. significantly increased with age $(p<0.001)$ but the percentage of memory CD8 ${ }^{+}$cells $\mathbf{D}$. was unaffected by age. Both parameters were unaffected by maternal diet. The naive:memory CD4 ${ }^{+} \mathbf{E}$. and $\mathrm{CD} 8^{+}(\mathrm{F})$ ratios were both affected by maternal diet $(p$ $<0.05)$ especially at $3 \mathrm{~m}$ of age $\left({ }^{*} p<0.05\right.$ and $* * p<0.01$ respectively). This ratio also significantly decreased with age in both cell types $(p$ $<0.001$ and $p<0.01$ respectively). $* p<0.05, * * p<0.01, * * * p<0.001 . n=5-9$ per group. Data are represented as mean $+/-$ SEM. 
lymphocytes, cells that initiate adaptive immune responses against peripheral antigens. Therefore both organs play an integral part in functional immunity. In rodents and humans the development of the thymus takes place during the late fetal / early postnatal stages of growth, whereas the development of the spleen begins earlier in gestation $[23,25,26]$. Given this, both organs may be vulnerable to early life events. Previous evidence suggests that the thymus and spleen are affected by nutritional insults in early life $[6,13,27,28]$. We therefore hypothesised that protein restriction during lactation which results in slowed growth and extended longevity may influence thymic and splenic function throughout life.

The present study firstly investigated changes in thymic function in control and PLP animals with increasing age. We observed a decrease in thymic
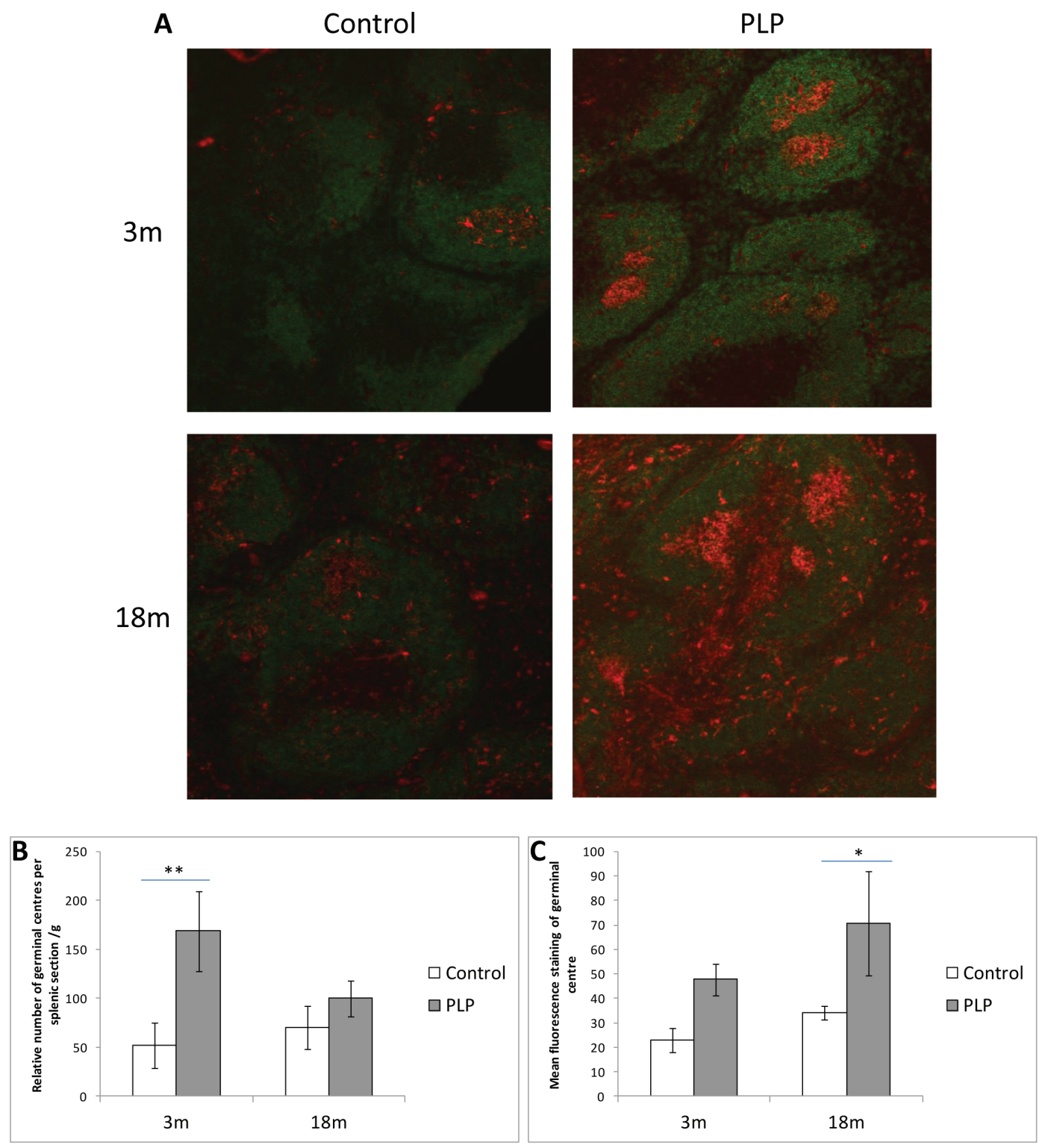

Figure 5: Effect of age and maternal diet on splenic B cell and germinal centre staining. A. Sections of spleen were fixed in acetone and incubated in PNA biotin conjugate (Vector Laboratories, Peterborough) followed by Streptavidin Alexa Fluor 594 conjugate (Invitrogen, Molecular Probes, Paisley) in order to stain for GCs (red). Double staining was performed where sections were then incubated in a FITC labelled polyclonal anti-B220 antibody (eBioscience). Following staining of 4 sections per animal representing different crosssections across the spleen, slides were mounted in Vectashield Mounting Medium (Vector laboratories) and visualised on the Zeiss LSM 510 META confocal microscope using Zen 2008 software. B. The number of GCs per section was recorded and represented as a proportion of splenic weight. PLP animals had a significantly higher relative density of GCs compared to controls $(p<0.05)$ and this was particularly apparent at $3 \mathrm{~m}$ of age $(* * p<0.01)$. C. The mean staining intensity of GCs was recorded and was found to be significantly higher in PLP animals compared to controls $(p<0.01)$, particularly at $18 \mathrm{~m}\left({ }^{*} p<0.05\right)$. However there was no significant effect of age on either parameter. $n=6-8$ per group. Data are represented as mean $+/-$ SEM. 
cellularity in old age, indicative of thymic involution. However PLP offspring demonstrated greater thymic cellularity with a greater number of $\mathrm{T}$ cells compared to controls. We previously demonstrated that PLP animals had a greater increase in thymic weight between weaning and adulthood compared to control animals [13]. Altogether these parameters suggest a delay in thymic involution in PLP animals. Given the extended longevity observed in these animals, and that thymic involution is a robust biomarker of aging, this suggests a slower rate of thymic aging in the PLP group.

In thymocyte development, pre-TCR expression and signalling occurs at the DN3 stage and progressively increases so that CD3 expression is highest in the most mature thymocytes $[16,17]$. The mean intensity of CD3 expression on thymocytes decreased in old age, and this is consistent with previous studies [29]. The age-associated decrease in mean intensity of CD3 expression on SP CD4 ${ }^{+}$ thymocytes was significantly less marked in PLP animals compared to controls. Given the crucial role of CD3 in the TCR transduction signalling pathway, a decrease in CD3 expression is likely to negatively impact upon responses to TCR-dependent signals and hence impair thymopoiesis [30]. These results show that PLP animals better maintain $\mathrm{CD} 3$ expression on $\mathrm{CD}^{+}$thymocytes than control animals throughout the life course and could suggest improved TCR signalling and $\mathrm{T}$ cell responsiveness in these animals. Here there is evidence that diet in the early postnatal period can modulate the aging trajectory of the immune system and shows protection against the effects of aging.

There were a number of thymic parameters that were only affected by age. The proportion of DN1 thymocytes increased with age whereas the proportion of DN2 thymocytes decreased with age suggesting a developmental block between these stages, again consistent with previous studies [31, 32]. During this stage diversity-joining (D-J) gene rearrangement of the TCR $\beta$ chain occurs which may be impaired with age [33]. The observed decrease in the proportion of DN4 thymocytes in old age has also been described previously [34]. During this stage mass proliferation and upregulation of CD4 and CD8 receptors occurs, which may also be subsequently impaired with increasing age [17].

Given the observed changes in the thymus, the secondary lymphoid tissue, the spleen was investigated. Splenic development peaks at puberty which is followed by gradual involution throughout life [23]. As reported in previous studies, relative splenic cellularity decreased with age [35]. However relative splenic cellularity was increased at each time point in the PLP group suggesting a slower rate of splenic aging in these animals. Although recent thymic emigrants are fewer in number and diversity with increasing age, homeostatic mechanisms are in place to ensure a constant size of the peripheral $\mathrm{B}$ and $\mathrm{T}$ cell pool [20]. Therefore PLP animals are likely to require less clonal $\mathrm{T}$ cell expansion in order to maintain the same peripheral $\mathrm{T}$ cell numbers with increasing age.

In advancing age fewer naive $\mathrm{T}$ cells are released from the thymus and so the number of memory $\mathrm{T}$ cells increase in the periphery. This results in the loss of TCR diversity in aged individuals and is further exacerbated by homeostatic clonal expansion [36]. Furthermore memory $\mathrm{T}$ cells dampen the thymic production of naive cells, thereby perpetuating the proportion of memory $\mathrm{T}$ cells in the periphery [37]. This phenomenon is responsible for the increased incidence of neoplasia and infection observed in the elderly. For example the shift in balance from naive to memory $\mathrm{T}$ cells is a mechanism that contributes towards the spontaneous incidence of Hodgkin's-like lymphoma in aged SJL/J mice [38]. We observed a significantly higher proportion of naive $\mathrm{CD}^{+}$and $\mathrm{CD}^{+} \mathrm{T}$ cells and a significantly higher ratio of naive:memory $\mathrm{CD}^{+}$and

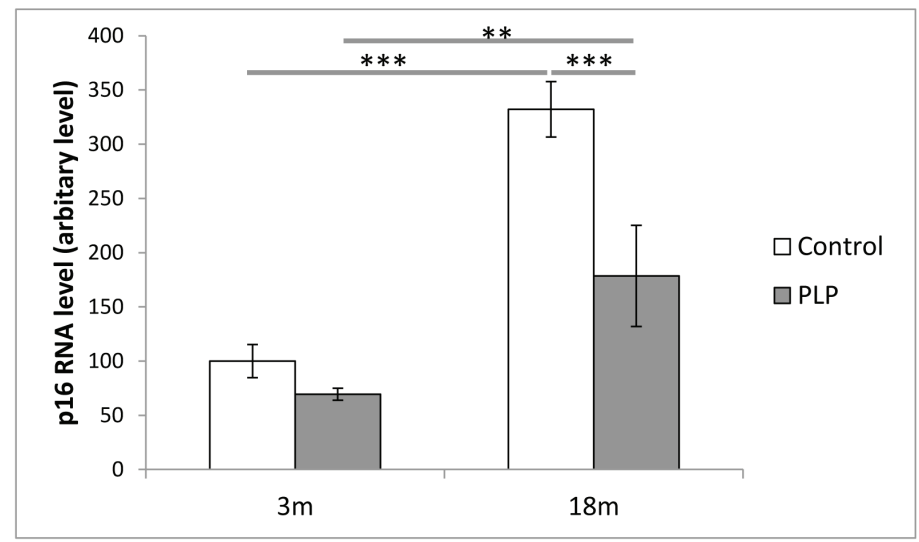

Figure 6: Effect of age and maternal diet on relative gene expression of $\mathbf{p} 16$ in the spleen. Gene expression levels were measured by quantitative RT-PCR. Values are expressed with mean gene expression in the spleen from $3 \mathrm{~m}$ control mice being set as 100 . Gene expression of p16 was significantly affected by both age $(p<0.001)$ and maternal diet $(p<0.001)$. However PLP animals had a less marked age-associated increase in expression compared to controls. ${ }^{* *} p<0.01,{ }^{* * *} p<0.001 . n=6-8$ per group. Data are represented as mean $+/$ - SEM. 
$\mathrm{CD}^{+} \mathrm{T}$ cells in PLP animals. This is likely due to the increased thymic cellularity observed in PLP animals and may suggest that PLP animals would be better protected from a pathogenic challenge and may be less susceptible to cancers. In order to directly determine the effect of maternal diet on $\mathrm{T}$ cell mediated immune responses, it would be necessary to infect animals with a viral challenge and measure differences in morbidity and mortality in animals.

The functioning of peripheral immune cells is greatly affected by splenic architecture which was therefore investigated on a histological level. GCs are sites of $B$ cell activation where naive $B$ cells enter and undergo clonal expansion and somatic hypermutation in the dark zone, followed by affinity maturation, $\mathrm{T}$ cell-and dendritic cell-dependent selection and class-switching in the light zone [39]. Following this, cells either generate into antibody secreting plasmablasts or form early memory B cells and enter the periphery [40]. Animals were kept in the same conditions with a low level of pathogens. Our results showed an increase in staining and density of GCs in PLP animals compared to control animals which may indicate increased B cell activity in these animals.

p16 is a senescence-promoting tumour suppressor protein that inhibits the cell cycle and has been linked to cancer and aging of nearly all mammalian tissues. It is not only associated with aging but does appear to play a causal role in some tissues [41]. The observed decrease in p16 gene expression in spleens from PLP animals suggests a slower rate of splenic aging in comparison to control animals, and indicates that maternal diet can better protect against age-associated alterations.

The developmental origins of health and disease hypothesis describes the phenomenon whereby the environment experienced in early life can influence the long-term health of the individual. The current findings support the hypothesis that as well as affecting metabolic parameters, early nutrition can impact upon immune function. The observed delay in aging of both the central and peripheral immune systems in animals exposed to reduced protein during the lactation period, may contribute to the increased lifespan observed in these animals. The suckling period may therefore represent a tractable period for intervention to reduce the burden of age-associated diseases.

\section{MATERIALS AND METHODS}

Mice

All procedures on animals were conducted under the British Animals (Scientific Procedures) Act (1986). $\mathrm{C} 57 \mathrm{BL} / 6$ mice were bred at a designated animal unit at University of Cambridge. They were housed at $22^{\circ} \mathrm{C}$ on a controlled $12 \mathrm{~h}$ light/dark cycle with access to standard laboratory chow and water. They were mated at 6 weeks of age and females were assumed to be pregnant when a vaginal plug was expelled. Dams were fed ad libitum either a control diet (20\% protein) or an isocaloric low protein diet ( $8 \%$ protein) during gestation and lactation. These diets were purchased from Arie Blok (Woerden, the Netherlands) and their compositions were described previously [22].

Two days after birth, cross fostering techniques were utilised to establish two groups: The control group (offspring born to and suckled by dams fed a control diet) and the PLP group (offspring of control dams cross-fostered and suckled by dams fed the low protein diet). In order to maximise the effect of maternal diet on differences in offspring postnatal growth control litters were culled to a size of 6 pups, whereas PLP pups were left unculled. Male offspring were fasted overnight and culled using $\mathrm{CO}_{2}$ at $21 \mathrm{~d}$ (weaning), $3 \mathrm{~m}$ (young adult), $18 \mathrm{~m}$ (old adult) and $23 \mathrm{~m}$ (severely old age). Fresh thymic and splenic tissues were immediately removed, weighed and used for flow cytometry analysis. Splenic tissues were snap-frozen in liquid nitrogen and stored at $-80^{\circ} \mathrm{C}$ until use.

\section{Flow cytometry analysis}

Cellular preparation and staining was performed as described previously [42]. Briefly thymi and spleen were teased apart and cell suspensions were collected in PBS. Splenic cells were incubated in red blood cell lysate before cellularity of both suspensions was quantified using a Countess Automated Cell Counter (Invitrogen, Paisley, UK). Cells were stained with FITC (fluorescein isothiocyanate)-, PE (phycoerythrin)- and PerCP-Cy5.5labelled polyclonal antibodies against the following markers: CD3, CD4 and CD8, and isotype controls (in both thymus and spleen cells); CD44, CD4, CD8 and CD25 (thymus cells); CD3 and B220 (spleen cells); CD44, CD4 and CD62L (spleen cells); and CD44, CD8 and CD62L (spleen cells). All antibodies were purchased from eBioscience (Hertfordshire, UK). Flow cytometry data were collected using a BD FACSCalibur flow cytometer and appropriate gating was performed. Data were expressed as the percentage of gated live lymphocytes which was determined according to forward and side scatter (FSC/SSC).

\section{Immunofluorescence}

Spleens were sectioned $(7 \mu \mathrm{m}$ thick), air dried overnight, fixed in acetone and stored at $-20^{\circ} \mathrm{C}$. Sections were stained as previously described [43]. To detect GCs, sections were incubated in PNA biotin conjugate (Vector Laboratories, Peterborough, UK) appropriately diluted in 
PNA buffer (10mM HEPES, 0.15M NaCl, 0.1 $\mathrm{mM} \mathrm{Ca}^{2+}$, $\mathrm{pH} 7.5$ ) for $1 \mathrm{~h}$ at room temperature. Following three washes in PBS, slides were incubated in Streptavidin Alexa fluor 594 conjugate (Invitrogen, Molecular Probes, Paisley, UK) appropriately diluted in PBS for $1 \mathrm{~h}$ in the dark at room temperature. Double staining was performed in order to detect $\mathrm{B}$ cell regions. Here sections were additionally incubated in rat polyclonal anti-mouse B220 FITC conjugate antibody (eBioscience, Hatfield, UK) diluted appropriately in PBS. Negative controls consisted of primary incubation with $0.5 \%$ BSA followed by isotype control antibodies with no staining detected.

Following staining of 4 sections per animal representing different cross-sections across the spleen, slides were mounted in Vectashield Mounting Medium (Vector Laboratories, Ltd. Peterborough, UK) and visualised on the Zeiss LSM 510 META confocal microscope using the software Zen 2008 (Zeiss, Cambridge, UK). 3 images were taken of each section in order to obtain representative staining. The number of GCs per section and the mean staining intensity were quantified (Zen 2008).

\section{Extraction of total RNA and cDNA synthesis}

Total RNA samples were prepared using Trizol reagent (Sigma), then purified using RNeasy Mini Kits (Qiagen) which included a DNase digestion step to eliminate potential contaminating DNA. These were then quantified using a NanoDrop ND-1000 (ThermoScientific). First strand cDNA was reversetranscribed from $1 \mu \mathrm{g}$ of total RNA using an ImProm-II Reverse Transcription System (Promega) with oligo(dT) as the primer, according to the manufacturer's protocol.

\section{Gene expression analysis by RT-PCR (real-time PCR)}

Quantitative RT-PCR was carried out using an ABI PRISM 7900 Sequence Detection System (Applied Biosystems) with a SYBR Green PCR Master Mix (Applied Biosystems) and gene-specific primers. Primers were custom-designed and synthesised by Sigma. For p16 the forward primer was CTTTGTGTACCGCTGGGAAC and the reverse was GCCGGATTTAGCTCTGCTCT. For Cyclophilin A the forward primer was CTTGCTGCAGCCATGGTCAA and the reverse was GTCTGCAAACAGCTCGAAGG. cDNA template $(3 \mu \mathrm{l}$; diluted according to the relative expression level of the gene of interest) was used in a $12 \mu$ l total reaction volume in each well in a 96-well reaction plate. A dissociation curve analysis was also performed to ensure primers did not form primer dimers. The transcripts were amplified in duplicate and standard curves were drawn using serially diluted pooled cDNA samples from each animal. Relative expression levels were calculated against the gene's standard curve with the $\mathrm{C}_{\mathrm{t}}$ values of each animal, using the housekeeping gene Cyclophilin A as a loading control. Cyclophilin A expression was equal between control and PLP animals at all ages.

\section{Statistical analysis}

A one-way or two-way ANOVA was performed using Statistica (StatSoft Ltd, Milton Keynes, UK) with maternal diet and age as the independent variables as appropriate. A Duncan's post-hoc test was used if appropriate and displayed on the figures and tables. A $p$ value of $<0.05$ was considered statistically significant.

\section{ACKNOWLEDGMENTS}

The authors are grateful to Adrian Wayman and Delia Hawkes for technical assistance.

\section{GRANT SUPPORT}

This work was supported by the BBSRC and the MRC. SEO is funded by the University of Cambridge MRC Metabolic Diseases Unit (MRC_MC_UU_12012/4).

\section{CONFLICTS OF INTEREST}

The authors have no financial or commercial conflicts of interest.

\section{REFERENCES}

1. Langley-Evans SC, Alexander B, McArdle HJ and Sloboda DM. Developmental origins of health and disease. Journal of nutrition and metabolism. 2012; 2012:838640.

2. Hales CN, Barker DJ, Clark PM, Cox LJ, Fall C, Osmond $\mathrm{C}$ and Winter PD. Fetal and infant growth and impaired glucose tolerance at age 64. BMJ. 1991; 303:1019-1022.

3. Eriksson JG, Forsen T, Tuomilehto J, Winter PD, Osmond $\mathrm{C}$ and Barker DJ. Catch-up growth in childhood and death from coronary heart disease: longitudinal study. BMJ. 1999; 318:427-431.

4. Dorner $\mathrm{G}$ and Plagemann A. Perinatal hyperinsulinism as possible predisposing factor for diabetes mellitus, obesity and enhanced cardiovascular risk in later life. Horm Metab Res. 1994; 26:213-221.

5. Fewtrell MS, Prentice A, Jones SC, Bishop NJ, Stirling D, Buffenstein R, Lunt M, Cole TJ and Lucas A. Bone mineralization and turnover in preterm infants at 8-12 years of age: the effect of early diet. J Bone Miner Res. 1999; $14: 810-820$.

6. Collinson AC, Moore SE, Cole TJ and Prentice AM. Birth season and environmental influences on patterns of thymic 
growth in rural Gambian infants. Acta Paediatr. 2003; 92:1014-1020.

7. Xu MQ, Sun WS, Liu BX, Feng GY, Yu L, Yang L, He G, Sham P, Susser E, St Clair D and He L. Prenatal malnutrition and adult schizophrenia: further evidence from the 1959-1961 Chinese famine. Schizophrenia bulletin. 2009; 35:568-576.

8. Lucas A. Long-term programming effects of early nutrition — implications for the preterm infant. J Perinatol. 2005; 25 Suppl 2:S2-6.

9. Fischer A, Le Deist F, Hacein-Bey-Abina S, Andre-Schmutz I, Basile Gde S, de Villartay JP and Cavazzana-Calvo M. Severe combined immunodeficiency. A model disease for molecular immunology and therapy. Immunological reviews. 2005; 203:98-109.

10. Moore SE, Cole TJ, Poskitt EM, Sonko BJ, Whitehead RG, McGregor IA and Prentice AM. Season of birth predicts mortality in rural Gambia. Nature. 1997; 388:434.

11. Moore S, Prentice A, Wagatsuma Y, Fulford A, Collinson A, Raqib R, Vahter M, Persson L and Arifeen S. Early-life nutritional and environmental determinants of thymic size in infants born in rural Bangladesh. Acta Paediatr. 2009; 98:1168-75.

12. Osgerby JC, Wathes DC, Howard D and Gadd TS. The effect of maternal undernutrition on ovine fetal growth. The Journal of endocrinology. 2002; 173:131-141.

13. Chen JH, Tarry-Adkins JL, Heppolette CA, Palmer DB and Ozanne SE. Early-life nutrition influences thymic growth in male mice that may be related to the regulation of longevity. Clin Sci (Lond). 2009; 118:429-438.

14. Ozanne SE and Hales CN. Lifespan: catch-up growth and obesity in male mice. Nature. 2004; 427:411-412.

15. Godfrey DI, Kennedy J, Suda $\mathrm{T}$ and Zlotnik A. A developmental pathway involving four phenotypically and functionally distinct subsets of CD3-CD4-CD8- triplenegative adult mouse thymocytes defined by CD44 and CD25 expression. J Immunol. 1993; 150:4244-4252.

16. Azzam HS, Grinberg A, Lui K, Shen H, Shores EW and Love PE. CD5 expression is developmentally regulated by $\mathrm{T}$ cell receptor (TCR) signals and TCR avidity. The Journal of experimental medicine. 1998; 188:2301-2311.

17. Carpenter AC and Bosselut R. Decision checkpoints in the thymus. Nature immunology. 2012; 11:666-673.

18. Gordon J and Manley NR. Mechanisms of thymus organogenesis and morphogenesis. Development (Cambridge, England). 2011; 138:3865-3878.

19. Aspinall R and Goronzy JJ. Immune senescence. Current opinion in immunology. 2010; 22:497-499.

20. Aw D, Silva AB, Maddick M, von Zglinicki T and Palmer DB. Architectural changes in the thymus of aging mice. Aging cell. 2008; 7:158-167.

21. Castle SC. Clinical relevance of age-related immune dysfunction. Clin Infect Dis. 2000; 31:578-585.

22. Chen JH, Martin-Gronert MS, Tarry-Adkins J and Ozanne
SE. Maternal protein restriction affects postnatal growth and the expression of key proteins involved in lifespan regulation in mice. PloS one. 2009; 4:e4950.

23. Cesta MF. Normal structure, function, and histology of the spleen. Toxicol Pathol. 2006; 34:455-465.

24. Cheung HT and Nadakavukaren MJ. Age-dependent changes in the cellularity and ultrastructure of the spleen of Fischer F344 rats. Mechanisms of ageing and development. 1983; 22:23-33.

25. Farley AM, Morris LX, Vroegindeweij E, Depreter ML, Vaidya H, Stenhouse FH, Tomlinson SR, Anderson RA, Cupedo T, Cornelissen JJ and Blackburn CC. Dynamics of thymus organogenesis and colonization in early human development. Development (Cambridge, England). 2013; 140:2015-2026.

26. Timens W, Rozeboom T and Poppema S. Fetal and neonatal development of human spleen: an immunohistological study. Immunology. 1987; 60:603-609.

27. Odaka Y, Nakano M, Tanaka T, Kaburagi T, Yoshino $\mathrm{H}$, Sato-Mito N and Sato K. The Influence of a High-Fat Dietary Environment in the Fetal Period on Postnatal Metabolic and Immune Function. Obesity (Silver Spring). 2010; 18:1688-94.

28. da Silva SV, Salama C, Renovato-Martins M, Helal-Neto E, Citelli M, Savino W and Barja-Fidalgo C. Increased leptin response and inhibition of apoptosis in thymocytes of young rats offspring from protein deprived dams during lactation. PloS one. 2013; 8:e64220.

29. Aw D, Silva AB and Palmer DB. The effect of age on the phenotype and function of developing thymocytes. Journal of comparative pathology. 2010; 142 Suppl 1:S45-59.

30. Ohashi PS, Pircher H, Burki K, Zinkernagel RM and Hengartner H. Distinct sequence of negative or positive selection implied by thymocyte T-cell receptor densities. Nature. 1990; 346:861-863.

31. Zhu X, Gui J, Dohkan J, Cheng L, Barnes PF and Su DM. Lymphohematopoietic progenitors do not have a synchronized defect with age-related thymic involution. Aging cell. 2007; 6:663-672.

32. Aspinall R and Andrew D. Thymic atrophy in the mouse is a soluble problem of the thymic environment. Vaccine. 2000; 18:1629-1637.

33. Krangel MS. Mechanics of $\mathrm{T}$ cell receptor gene rearrangement. Current opinion in immunology. 2009; 21:133-139.

34. Li L, Hsu HC, Grizzle WE, Stockard CR, Ho KJ, Lott P, Yang PA, Zhang HG and Mountz JD. Cellular mechanism of thymic involution. Scandinavian journal of immunology. 2003; 57:410-422.

35. Pinchuk LM and Filipov NM. Differential effects of age on circulating and splenic leukocyte populations in $\mathrm{C} 57 \mathrm{BL} / 6$ and BALB/c male mice. Immun Ageing. 2008; 5:1.

36. Nikolich-Zugich J and Rudd BD. Immune memory and aging: an infinite or finite resource? Current opinion in 
immunology. 2010; 22:535-540.

37. Surh CD and Sprent J. Homeostasis of naive and memory $T$ cells. Immunity. 2008; 29:848-862.

38. Han GM, Zhao B, Jeyaseelan S and Feng JM. Ageassociated parallel increase of Foxp3(+)CD4(+) regulatory and $\mathrm{CD} 44(+) \mathrm{CD} 4(+)$ memory $\mathrm{T}$ cells in $\mathrm{SJL} / \mathrm{J}$ mice. Cellular immunology. 2009; 258:188-196.

39. Allen CD, Okada $\mathrm{T}$ and Cyster JG. Germinal-center organization and cellular dynamics. Immunity. 2007; 27:190-202

40. Blink EJ, Light A, Kallies A, Nutt SL, Hodgkin PD and Tarlinton DM. Early appearance of germinal center-derived memory B cells and plasma cells in blood after primary immunization. The Journal of experimental medicine. 2005; 201:545-554.

41. Liu Y, Johnson SM, Fedoriw Y, Rogers AB, Yuan $\mathrm{H}$, Krishnamurthy $\mathrm{J}$ and Sharpless NE. Expression of p16(INK4a) prevents cancer and promotes aging in lymphocytes. Blood. 2011; 117:3257-3267.

42. Solomou K, Ritter MA and Palmer DB. Somatostatin is expressed in the murine thymus and enhances thymocyte development. European journal of immunology. 2002; 32:1550-1559.

43. Silva AB, Aw D and Palmer DB. Evolutionary conservation of neuropeptide expression in the thymus of different species. Immunology. 2006; 118:131-140. 